Ciência Florestal, Santa Maria, v. 21, n. 2, p. 355-362, abr.-jun., 2011

ISSN 0103-9954

\title{
SOBREVIVÊNCIA E CRESCIMENTO INICIAL DE MUDAS DE Pinus taeda L. COM DIFERENTES DIÂMETROS DO COLO
}

\author{
SURVIVAL AND INITIAL GROWTH OF Pinus taeda L. SEEDLINGS WITH DIFFERENT \\ COLLAR DIAMETERS
}

Raúl Vicente Pezzutti ${ }^{1}$ Silvana Lucia Caldato ${ }^{1}$

RESUMO

Com o objetivo de avaliar a sobrevivência e o crescimento de mudas de Pinus taeda produzidas em recipientes e em raiz nua com diferentes diâmetros do colo (DC) inicial, foram estabelecidos dois experimentos em solo de drenagem deficiente localizados na província de Corrientes, Argentina. O delineamento experimental utilizado foi o de blocos completos ao acaso, com três repetições por tratamento, sendo cada unidade experimental composta por 64 plantas e bordadura perimetral dupla. Os tratamentos avaliados, no experimento 1, foram mudas de recipientes com DC de 1,6 mm; $2 \mathrm{~mm} ; 3 \mathrm{~mm}$ e $4 \mathrm{~mm}$, e no experimento 2, foram mudas de raiz nua com DC de $3 \mathrm{~mm} ; 4 \mathrm{~mm} ; 5 \mathrm{~mm}$ e $6 \mathrm{~mm}$. Aos 4 anos de idade, as mudas de recipientes não apresentaram diferenças significativas em sobrevivência e crescimento; enquanto que nas mudas de raiz nua, a sobrevivência foi maior nos tratamentos de 4 e $5 \mathrm{~mm}$ não se encontrando diferença significativa em crescimento entre os tratamentos. Para os dois tipos de produção de mudas, o diâmetro do colo não teve influência significativa no crescimento a campo.

Palavras-chave: desenvolvimento; qualidade de muda; parâmetros morfológicos.

\begin{abstract}
The aim of this research was to evaluate the survival and growth of Pinus taeda seedlings produced in containers and bare root with different collar diameters. Two trials were established in a poor drained area located in the Corrientes province, Argentina. The experimental design was randomized complete blocks, with 3 replications and experimental units with 64 seedlings with double lines buffer. The treatments evaluated on the trial 1 were seedlings produced in containers with collar diameter of $1,6 \mathrm{~mm} ; 2 \mathrm{~mm} ; 3 \mathrm{~mm}$ and $4 \mathrm{~mm}$ and on the trial 2 were bare root seedlings with collar diameters of $3 \mathrm{~mm} ; 4 \mathrm{~mm} ; 5 \mathrm{~mm}$ and 6 $\mathrm{mm}$. At 4-year-old the seedlings produced in containers did not showed significant differences in survival and growth however in the bare root seedlings production, the seedlings with 4 and $5 \mathrm{~mm}$ showed the best survival. No significant differences in growth were detected for all the treatments. For both types of seedling production the collar diameter did not showed significant influence in field growth.
\end{abstract}

Keywords: development; seedling quality; morphological parameters.

\section{INTRODUÇÃO}

As mudas são insumos básicos na cadeia produtiva do setor florestal. O sucesso da plantação começa com a qualidade das mudas cujas características morfológicas e fisiológicas estão associadas com crescimento e sobrevivência favoráveis em diferentes condições de sítios (MATTSSON, 1996; WARD et al.,2000;
DAVIS e JACOBS, 2005). Segundo Duryea (1984), mudas de alta qualidade são aquelas que atingem níveis definidos de crescimento e sobrevivência para um sítio determinando. Para Maclaren (1993), a planta jovem que vai para o campo é um produto de tecnologia avançada, na qual a qualidade é importante para alcançar altos níveis de sobrevivência e rápido crescimento inicial.

1. Engenheiro Florestal, Doutorando do Programa de Pós-graduação em Engenharia Florestal, Centro de Ciências Rurais, Universidade Federal de Santa Maria, Av. Roraima, 1000, CEP 97105-900, Santa Maria (RS). raulpezzutti@yahoo.com.br/scaldato@yahoo.com.br

Recebido para publicação em 18/05/2009 e aceito em 26/08/2010 
Segundo Carneiro (1995), o aumento da porcentagem de sobrevivência das mudas e o maior desenvolvimento destas após o plantio, influem na redução de custos de replantio e de manutenção. Tais resultados favoráveis podem ser obtidos utilizando mudas de melhor padrão de qualidade. Nesse sentido Rose e Hasse (1995) acrescentam que, a cada ano, as operações florestais privadas tentam aumentar o crescimento nos primeiros anos, não só para melhorar o retorno financeiro de longo prazo, diminuir as rotações e manter a sustentabilidade, mas também para melhorar o ganho econômico do curto prazo por meio da captura rápida do sítio esperando usar menores quantidades de agroquímicos.

Diversos autores apresentam parâmetros da planta ideal para o estabelecimento de plantios em determinadas condições ambientais e espécies (ROSE et al., 1990; MACLAREN, 1993; ROSE e HASSE, 1995; SCHULTZ, 1997; ESCOBAR, 2005). Existem parâmetros morfológicos e fisiológicos para caracterizar a qualidade da muda para plantios florestais, normalmente são utilizados os primeiros pela facilidade de observação e medição. Em geral, o diâmetro do colo (DC) é definido por meio de um valor mínimo e a altura por uma amplitude entre um valor máximo e um valor mínimo desta.

Autores como Thompson (1985); Schultz (1997) e Barnett et al. (2002) afirmam que o diâmetro do colo é uma variável de fácil avaliação e muito correlacionada com o crescimento após o plantio para Pinus taeda e outras espécies do mesmo gênero. O diâmetro da muda é de fácil medição, mostra uma aproximação da seção transversal do transporte da água, da resistência mecânica, e da capacidade relativa para tolerar altas temperaturas na superfície do solo (CLEARY et al., 1977 apud BIRCHLER et al., 1998). Rose e Hasse (1995) consideram que o DC é uma característica de qualidade comumente observada a qual possui uma correlação positiva ( $\mathrm{r}$ $=0,68)$ com o volume radicular. Para Rose, et al. (1990) o DC está intimamente relacionado ao vigor da muda, e os caules com maiores diâmetros tendem a ter maiores gemas, as quais possuem um elevado número de primórdios foliares pré-formados que se desenvolveram para ser os primeiros brotos de crescimento após o plantio.

Segundo Ritchie e Landis (2008), numerosos estudos mostram que o DC é o melhor indicador do desempenho após o plantio e, consequentemente, da qualidade da muda e que estes resultados foram utilizados para definir os graus de qualidade das mudas. Como tais definições variam conforme as condições dos sítios, os graus de qualidade deverão ser desenvolvidos para cada espécie e sitio específico.

Tanto os produtores de mudas quanto os silvicultores, ao tratar a qualidade da muda, consideram: a sanidade, o diâmetro do colo, a altura da muda, o desenvolvimento do sistema radicular, a lignificação do caule e o material genético como as principais características. Landis et al. (1994) apresentaram as especificações morfológicas normalmente usadas por produtores e usuários, indicando que a altura e o diâmetro do colo são as mais utilizadas. Em geral, produzir mudas de maior DC tem maiores custos pela relação que essa variável possui com o tamanho do recipiente, a densidade de mudas $/ \mathrm{m}^{2}$ e o tempo de produção no viveiro. Porém, maiores custos podem ser traduzidos em ganhos quando estes se associam a uma qualidade da muda que leve a obtenção de maiores sobrevivência e crescimento posterior no campo.

No nordeste da Argentina, poucos estudos podem ser encontrados definindo quais são os parâmetros ideais a serem utilizados em mudas de Pinus taeda, assim como estudos que apresentem resultados de sobrevivência e crescimento de mudas produzidas com diferentes parâmetros morfológicos e ou fisiológicos.

O objetivo do presente trabalho foi avaliar o crescimento de mudas de Pinus taeda produzidas em recipientes e em raiz nua com diferentes diâmetros do colo ao momento da realização do plantio.

\section{MATERIAL E MÉTODO}

\section{Localização e caracterização do sitio}

$\mathrm{O}$ presente estudo foi realizado em áreas da empresa Forestal Bosques del Plata S.A. (BDP) localizada na província de Corrientes, Argentina. As coordenadas geográficas da área de estudo são: $27^{\circ}$ $56^{\prime} 05^{\prime \prime}$ de Latitude Sul e $56^{\circ} 08^{\prime} 36^{\prime \prime}$ de Longitude Oeste. A altitude é de aproximadamente 93 m s.n.m. De acordo ao tipo de relevo as áreas são planas e com drenagem deficiente apresentando água na superfície do solo.

O clima da região segundo a classificação de Köppen é do tipo Cfa, sendo um clima mesotérmico, sem estação seca, com precipitação máxima no outono e verões quentes. A temperatura média anual é de $20,5^{\circ} \mathrm{C}$ e a precipitação média anual de 1867 $\mathrm{mm}$.

De acordo com Escobar (1996), o solo da região é de textura franco siltosa, com drenagem 
imperfeito, fortemente ácido e com elevada saturação de alumínio, cor escura e com concreções de ferro e manganês. A profundidade efetiva do solo é de $50 \mathrm{~cm}$. Conforme a classificação do Soil Taxonomy (USDA, 1999), o solo corresponde à ordem dos Inceptisoles.

\section{Produção das mudas}

As mudas de recipientes foram produzidas no viveiro "El Pindó" pertencente à empresa BDP. Os recipientes utilizados foram bandejas de 40 cavidades com uma capacidade volumétrica de $93 \mathrm{~cm}^{3}$ por cavidade. O sistema de produção foi o utilizado pela empresa para a produção operativa, considerando-se somente casca de Pinus sp. compostada como substrato e fertiirrigação conforme o estádio de desenvolvimento da muda. Combinações de N, P, K, Ca, Mg e $\mathrm{S}+$ Micronutrientes foram geradas e aplicadas conforme o tamanho da muda. Após a semeadura a solução usada na irrigação possuiu uma relação $\left(\mathrm{N}: \mathrm{P}_{2} \mathrm{O}_{5}: \mathrm{K}_{2} \mathrm{O}: \mathrm{CaO}: \mathrm{MgO}: \mathrm{SO}_{4}\right)$ de 1:1:1,3:0,3:0,8:1 aplicada durante 5 semanas; logo a relação foi de 1:0,9:0,6:0,3:0,6:0,8 por 10 semanas; seguida da relação $1: 1,1: 1,6: 0,4: 0,8: 1$ por 10 semanas mais, e por último a relação de 1:0:0:0,8:1,2:1,6 durante 7 semanas, completando 8 meses de produção. Em cada aplicação da solução foi verificada a condutividade elétrica, sendo mantida em torno a $1 \mu \mathrm{S} \mathrm{cm} \mathrm{cm}^{-1}$. A irrigação no viveiro foi realizada procurando manter o substrato em condição de capacidade de campo evitando excesso de umidade assim como o secado do substrato.

Para estabelecer o experimento, as mudas de raiz nua foram compradas no viveiro "La Forzada" pertencente a um produtor florestal da região. A densidade final (mudas de Pinus taeda $/ \mathrm{m}^{2}$ de solo) foi aproximadamente de 280 mudas $/ \mathrm{m}^{2}$. As plantas foram selecionadas pelo produtor conforme a descrição dos tratamentos solicitados e retiradas dos canteiros para serem transportadas e plantadas no campo durante as 24 horas posteriores à retirada.

Tanto na produção de mudas de recipientes como na produção de mudas de raiz nua, não foi realizada poda aérea, e a seleção dos diferentes tamanhos foi realizada procurando mudas com as dimensões definidas dentro da mesma área de produção, ou canteiro. As mudas foram selecionadas conforme o diâmetro do colo definido para cada tratamento utilizando-se um calibre de medição. As mudas apresentaram boa sanidade, sem deformações, sendo vigorosas e com um desenvolvimento radicular completo.

O material genético utilizado para a produção de mudas em recipientes foi Pinus taeda de procedência Marion County, proveniente de sementes de uma Área Produtora de Sementes regional. Nas mudas produzidas no sistema de raiz nua, o material genético utilizado foi de sementes de Pinus taeda da procedência Marion County, proveniente de um pomar clonal de sementes de primeira geração dos Estados Unidos.

As mudas foram irrigadas e transportadas imediatamente nas bandejas para o campo. No caso de raiz nua, as mudas foram transportadas em caixas plásticas específicas, com gel hidratado cobrindo as raízes, para a conservação da umidade até o momento do plantio. Tanto as mudas em bandejas como as transportadas em caixas plásticas foram enviadas para o campo em veículo fechado e acondicionado, cuidando de minimizar o impacto por vento ou temperatura elevada.

\section{Tratamentos}

\section{Experimento 1:}

Os quatro tratamentos avaliados correspondem à mudas produzidas em recipientes com os seguintes diâmetros do colo (mm): T1) 1,6 mm; T2) $2 \mathrm{~mm}$; T3) $3 \mathrm{~mm}$ e T4) $4 \mathrm{~mm}$.

\section{Experimento 2:}

Os quatro tratamentos avaliados foram mudas produzidas no sistema de raiz nua, com os seguintes diâmetros do colo: T1) $3 \mathrm{~mm}$; T2) 4 mm; T3) $5 \mathrm{~mm}$ e T4) $6 \mathrm{~mm}$.

Para os dois experimentos foram selecionadas mudas sadias com um desenvolvimento radicular completo. Os valores de altura das mudas não foram determinados ao estabelecimento do experimento, em geral foram observadas maiores alturas com os maiores diâmetros para cada tipo de muda.

\section{Delineamento experimental}

Nos dois experimentos estabelecidos, o delineamento experimental utilizado foi o de blocos completos ao acaso (GOMES, 1990), com três repetições por tratamento, sendo cada unidade experimental composta por 64 plantas (8 linhas $\mathrm{x}$ 8 plantas). As parcelas apresentaram bordadura perimetral dupla. 
Na Figura 1, apresenta se o croqui do experimento para mudas produzidas em recipientes e mudas produzidas em raiz nua.

\begin{tabular}{|c|c|c|}
\hline T3 & T3 & T4 \\
\hline T2 & T1 & T3 \\
\hline T4 & T2 & T2 \\
\hline T1 & T4 & T1 \\
\hline Bloco 3 & Bloco 2 & Bloco 1 \\
\hline
\end{tabular}

Experimento 1 Mudas produzidas em recipientes.

Tratamentos (Diâmetro do colo das mudas): T1: $1,6 \mathrm{~mm}$; T2: $2 \mathrm{~mm}$; T3: $3 \mathrm{~mm}$; e T4: $4 \mathrm{~mm}$.

\begin{tabular}{|c|c|c|c|}
\hline T1 & T3 & T4 & Experimento 2 - \\
\hline T4 & T1 & T3 & $\begin{array}{l}\text { raiz nua. } \\
\text { raidas em }\end{array}$ \\
\hline T3 & T4 & $\mathrm{T} 2$ & Tratamentos (Diân \\
\hline $\mathrm{T} 2$ & T2 & T1 & $\begin{array}{l}\text { colo das mudas): } \\
\text { T1: } 3 \mathrm{~mm} ; \mathrm{T} 2: 4 \mathrm{~mm} ; \mathrm{T}\end{array}$ \\
\hline Bloco 3 & Bloco 2 & Bloco 1 & $5 \mathrm{~mm}$ e T4: $6 \mathrm{~mm}$ \\
\hline
\end{tabular}

FIGURA 1: Croqui dos experimentos em delineamento de blocos ao acaso.

FIGURE 1: Layout of the trials in randomized blocks design.

\section{Plantio e condução do experimento}

$\mathrm{Na}$ área onde foram estabelecidos os experimentos, o solo foi preparado 3 meses antes da realização do plantio, realizando-se camalhões como modalidade do preparo. O plantio foi realizado manualmente com pá no dia 25/06/2003, o espaçamento utilizado foi de $4 \mathrm{~m}$ entre linhas $\mathrm{x}$ $1,75 \mathrm{~m}$ entre mudas da linha de plantio. Durante os 2 primeiros anos foram realizados controles químicos de ervas daninhas no linho do plantio $(1,8 \mathrm{~m}$ de largura), e os controles de formigas foram realizados periodicamente com formicidas (sulfluramida em iscas e deltametrina em pó).

\section{Medições realizadas e análises dos dados}

As medições foram efetuadas aos 4 anos de idade. As alturas totais foram medidas com clinómetro e o diâmetro a altura do peito foi medido com fita diamétrica. Foram quantificadas as plantas vivas e mortas durante a medição com a finalidade de determinar a sobrevivência de cada um dos tratamentos avaliados. Para o cálculo do volume por planta, foi utilizada uma função utilizada normalmente na empresa para esta espécie.

Foram estimadas as médias por planta em cada parcela para as variáveis, diâmetro à altura do peito (DAP) altura total (h) e volume. Os valores de sobrevivência foram transformados para raiz quadrada arcoseno para a análise (GOMES, 1990; STORCK, et al., 2000). Os dados foram submetidos à análise da variância. Quando foi verificada diferença significativa entre tratamentos foi realizado o teste de separação de medias de Tukey $(\mathrm{P}<0,05)$ para a avaliação destes.

\section{RESULTADO E DISCUSSÃO}

No experimento 1 (mudas em recipiente) aos 4 anos de idade, para as variáveis diâmetro à altura do peito (DAP), altura total (h), volume por planta e sobrevivência, os tratamentos avaliados não apresentaram diferenças significativas pelo teste de F.

$\mathrm{Na}$ Tabela 1, apresentam-se os valores médios e coeficientes de variação de diâmetro à altura do peito, altura total, volume por planta e percentagem de sobrevivência, para os tratamentos de mudas com diferentes diâmetros do colo inicial.

Os valores médios apresentados na Tabela 1 apontam, em geral, valores crescentes à medida que os DC são maiores, porém as diferenças observadas

TABELA 1: Valores médios de crescimento e sobrevivência de Pinus taeda aos 4 anos de idade, por tratamento, no experimento 1 ( mudas produzidas em recipientes).

TABLE 1: Growth and survival mean values of Pinus taeda at 4-year-old, by treatment, in the trial 1 (seedlings produced in containers).

\begin{tabular}{ccccc}
\hline $\begin{array}{c}\text { Tratamento } \\
(\mathrm{DC} \mathrm{mm})\end{array}$ & $\begin{array}{c}\mathrm{DAP} \\
(\mathrm{cm})\end{array}$ & $\begin{array}{c}\mathrm{H} \\
(\mathrm{m})\end{array}$ & $\begin{array}{c}\text { Volume } \\
\left(\mathrm{m}^{3} \text { planta }^{-1}\right)\end{array}$ & $\begin{array}{c}\text { Sobrevivência } \\
(\%)\end{array}$ \\
\hline $\begin{array}{c}\mathrm{T} 1 \\
(1,6 \mathrm{~mm})\end{array}$ & 11,6 & 6,0 & 0,0295 & 78,1 \\
$\mathrm{~T} 2$ & 11,8 & 6,3 & 0,0320 & 84,9 \\
$(2,0 \mathrm{~mm})$ & & & & \\
$\mathrm{T} 3$ & 11,9 & 6,0 & 0,0314 & 85,9 \\
$(3,0 \mathrm{~mm})$ & & & & \\
$\mathrm{T} 4$ & 12,2 & 6,5 & 0,0356 & 91,7 \\
$(4,0 \mathrm{~mm})$ & & & & 85,1 \\
\hline Média & 11,9 & 6,2 & 0,0321 & 16,91 \\
CV \% & 3,2 & 5,5 & 11,7 & \\
\hline
\end{tabular}

Em que: $\mathrm{DC}=$ diâmetro do colo; $\mathrm{DAP}=$ diâmetro à altura do peito; $\mathrm{H}=$ altura; $\mathrm{CV}=$ coeficiente de variação; ${ }^{1} \mathrm{CV}$ correspondente à variável transformada na analise. 
não foram detectadas como significativas no experimento para nenhuma das variáveis avaliadas. Diferenças de pequena magnitude são observadas nas variáveis de DAP e h. No caso da sobrevivência, a não diferenciação entre tratamentos pode ser por causa da maior variação (CV \%) existente nessa variável. As causas prováveis dessa maior variação podem ter sido a variação de umidade em microssítios, gerando condições não adequadas de excesso de umidade e afetando a sobrevivência das mudas e/ou ataques pontuais de formigas (ou outros insetos) embora fossem realizados controles de formigas de maneira mensal após o plantio.

No experimento 2 (mudas de raiz nua) aos 4 anos de idade, os tratamentos avaliados não diferiram significativamente pelo teste de $\mathrm{F}$ para as variáveis diâmetro à altura do peito, altura total e volume. A sobrevivênciaapresentou diferenças significativas pelo teste de $\mathrm{F}(\mathrm{P}<0,01)$.

$\mathrm{Na}$ Tabela 2, apresentam-se os valores médios e coeficientes de variação para as varáveis DAP, h, volume por planta e sobrevivência para os tratamentos de mudas com diferentes diâmetros do colo inicial e o resultado do teste de Tukey para a variável sobrevivência.

TABELA 2: Valores médios de crescimento, sobrevivência e teste de Tukey $(\mathrm{P}<$ $0,05)$ para a variável sobrevivência de Pinus taeda aos 4 anos de idade, por tratamento no experimento 2 .

TABLE 2: Growth and survival mean values, and Tukey test $(\mathrm{P}<0,05)$ for survival variable of Pinus taeda at 4-year-old by treatment in trial 2 .

\begin{tabular}{cccccc}
\hline $\begin{array}{c}\text { Tratamento } \\
(\mathrm{DC} \mathrm{mm})\end{array}$ & $\begin{array}{c}\mathrm{DAP} \\
(\mathrm{cm})\end{array}$ & $\begin{array}{c}\mathrm{H} \\
(\mathrm{m})\end{array}$ & $\begin{array}{c}\text { Volume } \\
\left(\mathrm{m}^{3} \text { planta }^{-1}\right)\end{array}$ & $\begin{array}{c}\text { Sobrevivência } \\
(\%)\end{array}$ \\
\hline $\begin{array}{c}\mathrm{T} 1 \\
(3 \mathrm{~mm})\end{array}$ & 11,4 & 5,9 & 0,0283 & 66,1 & $\mathrm{a}$ \\
$\mathrm{T} 2$ & 11,6 & 6,0 & 0,0296 & 81,2 & $\mathrm{~b}$ \\
$(4 \mathrm{~mm})$ & & & & & \\
$\mathrm{T} 3$ & 11,5 & 6,1 & 0,0295 & 87,5 & $\mathrm{~b}$ \\
$(5 \mathrm{~mm})$ & & & & & \\
$\mathrm{T} 4$ & 11,5 & 5,9 & 0,0290 & 66,7 & $\mathrm{a}$ \\
$(6 \mathrm{~mm})$ & & & & & \\
\hline $\begin{array}{c}\text { Média } \\
\text { CV \% }\end{array}$ & 11,5 & 6,0 & 0,029 & 75,4 \\
\hline
\end{tabular}

Em que: Em que: $\mathrm{DC}=$ diâmetro do colo; $\mathrm{DAP}=$ diâmetro à altura do peito; $\mathrm{H}=$ altura; $\mathrm{CV}=$ coeficiente de variação; ${ }^{1} \mathrm{CV}$ correspondente à variável transformada na analise.
As mudas, em geral e nos dois experimentos, apresentaram um bom crescimento. Valores semelhantes de crescimento aos 4 anos de idade para Pinus taeda foram encontrados por Pezzutti e Caldato (2004) para a região do nordeste da província de Corrientes na Argentina, em sítios úmidos com preparo do solo em camalhões.

Como foi apresentado nos parágrafos anteriores, os tratamentos de mudas produzidas em recipientes não apresentaram diferenças importantes nas variáveis avaliadas aos 4 anos de idade. Provavelmente, as técnicas adequadas de produção das mudas no viveiro (material genético homogêneo e de qualidade, adubação, irrigação, controle de ervas e substrato de qualidade) geraram mudas de diferentes tamanhos, mas sem deficiências em geral, as quais ao serem plantadas num sitio de boa qualidade com uma silvicultura adequada responderam de maneira similar em crescimento e sobrevivência.

Os autores Ritchie e Landis (2008) apresentam diversas situações ambientais nas quais mudas de maiores e menores DC tiveram melhor desempenho após o plantio. Tais diferenças marcadas ocorrem quando existem, no ambiente, fatores condicionantes do crescimento (neve, animais, vegetação competidora, ambientes muitos secos, temperaturas extremas).

Os resultados obtidos diferem dos apresentados por South et al. (2005) para mudas de Pinus palustris produzidas em recipientes com diferentes DC, pois os autores acharam, após duas estações de crescimento, uma correlação positiva entre o DC e a altura das árvores. Já Zida et al. (2008) examinando a qualidade inicial de mudas de duas espécies florestais na África, Acacia macrostaachya e Pterocarpus erinaceus, concluem que o tamanho inicial das mudas não afeta a sobrevivência e o crescimento no campo sendo esse resultado muito semelhante ao obtido no primeiro experimento do presente trabalho.

O diâmetro do colo como parâmetro mínimo de qualidade de mudas de Pinus sp. produzidas em recipientes, foi definido entre 2,5 e $3 \mathrm{~mm}$ por diversos autores (BARNETT e BRISETTE, 1986; LANDIS et al. 1994; RODRIGUEZ, 2001). Nos resultados apresentados para mudas de recipientes, pode-se observar que os valores de crescimento e sobrevivência obtidos ao plantar mudas com DC nessa classe são normais para a região, com valores médios mínimos de crescimento de 11,8 $\mathrm{cm}$ de DAP; 6,0 $\mathrm{m}$ de altura e $85 \%$ de sobrevivência aos 4 anos de idade. 
No experimento de mudas produzidas a raiz nua somente foram encontradas diferenças significativas na sobrevivência, sendo que os valores de DC intermédios (4 $\mathrm{mm}$ e $5 \mathrm{~mm}$ ) apresentaram os melhores resultados.

Os maiores valores obtidos correspondem aos tratamentos com diâmetro do colo de 4 e $5 \mathrm{~mm}$, sendo estes maiores num 15,1 e 21,4 \% em relação ao tratamento de $3 \mathrm{~mm}$ de DC, e num 14,5 e $20,8 \%$ em relação ao tratamento de $6 \mathrm{~mm}$ de diâmetro do colo. Schultz (1997) apresenta resultados de estudos realizados nos Estados Unidos mostrando que após um ano do plantio, povoamentos de Pinus taeda apresentaram 15 a $20 \%$ mais de sobrevivência quando as mudas de raiz nua tinham diâmetros a altura do colo entre $3,2 \mathrm{~mm} \mathrm{e} 4,8 \mathrm{~mm}$ quando comparadas com mudas de DC entre 1,6 e 2,4 mm. Também South e Mexal (1984) destacam que as mudas de raiz nua apresentaram melhor sobrevivência à medida que o DC foi maior, com valores inferiores a $60 \%$ quando o DC teve entre $1 \mathrm{e}$ $2 \mathrm{~mm}$ e superiores a $80 \%$ a partir de $4 \mathrm{~mm}$ chegando até $90 \%$ com DC de $6 \mathrm{~mm}$. Todavia, Schultz (1997) apresenta que pesquisas realizadas nos Estados Unidos mostram que, quando as condições após o plantio são adversas (falta de precipitação), mudas de maiores tamanhos podem apresentar menores sobrevivências.

Respostas muito variadas têm sido encontradas por diversos autores, num estudo apresentado por McGilvray e Barnett (1982), o diâmetro de mudas teve correlação positiva com a sobrevivência de Pinus palustris e não teve correlação para Pinus taeda e Pinus echinata.

Autores como Rose et al. (1990) afirmam que, no caso de mudas de raiz nua, o diâmetro do colo deve ser superior a $4 \mathrm{~mm}$ para Pinus taeda nos Estados Unidos, enquanto Rodriguez (2001) apresenta o mesmo valor para o nordeste da Argentina. Esses valores são coincidentes em parte com os obtidos no experimento 2, em que os melhores resultados são obtidos com mudas de $4 \mathrm{a}$ $5 \mathrm{~mm}$ de DC sendo recomendável trabalhar nessa classe de DC sem continuar aumentando o tamanho da muda.

Conforme Maclaren (1993), para a produção de mudas de qualidade de raiz nua de Pinus radiata, o diâmetro do colo deve ter valores entre 6 e 12 mm. Para Pinus palustris RodriguezTrejo e Duryea (2003) destacam que um indicador de qualidade para mudas produzidas em recipientes é um diâmetro do colo da raiz maior ou igual a 4,4 $\mathrm{mm}$ e, para raiz nua, de pelo menos $10 \mathrm{~mm}$. Esses valores são muito elevados em comparação aos normalmente usados no nordeste da Argentina. Nos resultados experimentais, pode-se observar que mudas produzidas em recipientes com um DC de $4 \mathrm{~mm}$ apresentaram uma sobrevivência de $92 \%$, e mudas produzidas a raiz nua com $5 \mathrm{~mm}$ de DC apresentaram uma sobrevivência de $87,5 \%$ aos 4 anos de idade, sendo estes valores adequados para a produção florestal.

Barnett et al. (2002) ressaltam que desenvolver padrões de qualidade requer a realização de plantios e a avaliação do desenvolvimento destes em diversos sítios e anos sucessivos. Estabelecer padrões de qualidade de mudas baseados em pesquisas é difícil porque mudas com padrões baixos em anos com abundante chuva podem sobreviver. Para Thompson (1985), a classificação por altura e DC é praticada por muitos viveiros para separar as mudas de baixa qualidade. Experimentos, que relacionam o tamanho da muda e o desempenho a campo, mostram resultados contraditórios. Em geral, quando o status fisiológico é igual, as mudas maiores crescem melhor, mas usualmente não sobrevivem tão bem quanto as de menor tamanho.

\section{CONCLUSÕES}

Com base nos resultados dos experimentos de Pinus taeda avaliados aos 4 anos de idade no nordeste da Argentina, chega-se às seguintes conclusões:

O diâmetro do colo inicial das mudas produzidas em recipientes não influencia significativamente o crescimento e a sobrevivência destas. Os valores médios de sobrevivência apresentam diferenças de até $13,6 \%$ entre os tratamentos com maior e menor DC inicial, sendo o valor médio do experimento de $85,1 \%$ e o valor máximo por tratamento de $91,7 \%$;

As mudas produzidas em recipientes apresentam um crescimento médio de $11,9 \mathrm{~cm}$ em DAP e de $6,2 \mathrm{~m}$ em altura total aos 4 anos de idade, sendo este um desenvolvimento normal para a região;

O diâmetro do colo inicial das mudas produzidas a raiz nua não influencia significativamente o crescimento delas;

As mudas de raiz nua com diâmetro do colo entre 4 e $5 \mathrm{~mm}$ apresentam a melhor sobrevivência com diferenças de até $21,4 \%$ entre os valores 
extremos, sendo o valor médio do experimento de $75,4 \%$ e o valor máximo por tratamento de $87,5 \%$. As mudas produzidas a raiz nua apresentam um crescimento médio de $11,5 \mathrm{~cm}$ em DAP e de 6,0 $\mathrm{m}$ em altura total aos 4 anos de idade, sendo este um desenvolvimento normal para a região.

\section{REFERÊNCIAS BIBLIOGRÁFICAS}

BARNETT, J. P.; BRISSETTE, J. C. Producing southern pine seedlings in containers. General Technical Report SO-59. New Orleans: USDA Forest Service, Southern Forest Experiment Station. 1986. $71 \mathrm{p}$.

BARNETT, J. P.; HAINDS, M.; HERNANDEZ, G. Interim guidelines for growing longleaf seedlings in containers. In: WORKSHOPS ON GROWING LONGLEAF PINE IN CONTAINERS, 2002, Asheville. Proceedings... General Technical Report SRS-56. Asheville, NC: U.S. Department of Agriculture, Forest Service, Southern Research Station. 2002. p. 27-29.

BIRCHLER, T. et al. La planta ideal: revisión del concepto, parámetros definitorios e implementación práctica. Investigación Agraria: Sistemas y Recursos Forestales, Madrid, v. 7, n 1/2, p. 109121, 1998.

CARneiro, J. G. A. Produção e controle de qualidade de mudas florestais. Curitiba: UFR/ FUPEF, 1995. $451 \mathrm{p}$.

DAVIS, A. S.; JACOBS, D. F. Quantifying root system quality of nursery seedlings and relationship to outplanting performance. New Forests, Dordrecht, v. 30, p. 295-311, 2005.

DURYEA, M. L. Nursery cultural practices: impacts on seedling quality. In: DURYEA, M. L.; LANDIS, T. D. (Eds.) Forest nursery manual: production of bareroot seedlings. Corvallis: Martinus Nilhoff/ Dr. W. Junk Publishers, The Hague/Boston/ Lancaster, for forest research Laboratory, Oregon State University. 1984. p. 143-164.

ESCOBAR, E. et al. Mapa de suelos de la provincia de Corrientes. Corrientes. INTA, 1996, 429 p.

ESCOBAR, R. Establecimiento de plantaciones: manual práctico de manejo para plantaciones de Pinus radiata. Santiago: Fundación Chile, 2005. p. 5-31.

GOMES, F. P. Curso de estatística experimental. 13. ed. Piracicaba: Nobel, 1990. 468 p.

LANDIS, T. D. et al. Nursery planning, development and management. In: LANDIS, T. D. et al. (Eds.) The container tree nursery manual. Washington:
U.S. Department of Agriculture, Forest Service, 1994. v.1, 188 p. (Agricultural Handbook 674)

McGILVRAY J. M.; BARNETT J. P. Relating seedling morphology to field performance of containerized southern pines. In: SOUTHERN CONTAINERIZED FOREST TREE SEEDLING CONFERENCE, 1982. Proceedings... USDA. Forest Service, General Technical Report SO-37. 1982. p. 39-46.

MACLAREN, J. P. Radiata pine growers' manual. New Zealand: New Zealand Forest Research Institute,1993.140 p.( FRI Bulletin, n.184)

MATTSSON, A. Predicting field performance using seedling quality assessment. New Forests, Dordrecht, v. 13, p. 223-248, 1996.

PEZZUTTI, R.; CALDATO, S. Efecto del control de malezas en el crecimiento de plantaciones de Pinus taeda, Pinus elliottii var. elliottii y Pinus elliottii var. elliottii $\mathrm{x}$ Pinus caribaea var. hondurensis. Bosque, Valdivia, v. 25, n.2, p. 77-87, 2004.

RITCHIE, G. A.; LANDIS, T. D. The container tree nursery manual. USDA, v. 7, p. 17-80. 2008. (Agricultural Handbook 674) Disponível em: <(http:// www.rngr.net/Publications/ctnm/ volume7)> Acesso em: 11 de abril de 2009.

RODRIGUEZ, F. El paquete tecnológico aplicado por Bosques del Plata S.A. In: JORNADAS FORESTALES DE ENTRE RÍOS, 16., 2001, Concordia. Anales... Concordia: INTA - AIANER, 2001. 28 p.

RODRÍGUEZ-TREJO, D. A.; DURYEA, M. L. Seedling quality indicators in Pinus palustris Mill. Agrociencia, México, v. 37, p. 299-307, mai./jun., 2003.

ROSE, R.; HASSE, D. L. The target seedling concept: Implementing a Program. In: LANDIS, T. D.; CREGG, B. (Eds.) Forest and Conservation Nursery Associations Proceedings. General Technical Report PNW-GTR-365. Portland, OR: U.S: Department of Agriculture, Forest Service, Pacific Northwest Research Station. 1995. p. 124130.

ROSE, R.; CARLSON, W.; MORGAN, P. The target seedling concept. In.: ROSE, R.; CAMPBELL, S. J.; LANDIS, T. D. (Eds.) Proceedings of Western Forest Nursery Association. Roseburg, OR. General Technical Report RM-200. Fort Collins, CO: U.S. Department of Agriculture, Forest Service, Rocky Mountain Forest and Range Experiment Station, 1990. p. 1-8.

SCHULTZ, R. Loblolly pine: the ecology and culture of loblolly pine (Pinus taeda L.). USDA 
Agricultural Handbook 713. Washington, DC: U.S. Department of Agriculture, Forest Service. 1997. SOUTH, D. B.; MEXAL, J. G. Growing the best seedling for reforestation success. Forest Service 12. Auburn, Alabama Agricultural Experiment Station. p. 4-11, 1984.

SOUTH, D. B. et al. Effect of container type and seedling size on survival and early height growth of Pinus palustris seedlings in Alabama, U.S.A. Forest Ecology and Management, Amsterdam, v. 204, p. 385-398, 2005.

STORCK, L. et al. Experimentação vegetal. Santa Maria: Editora UFSM, 2000. 198 p.

THOMPSON, B. E. Seedling morphological evaluation - what you can tell by looking. In: DURYEA, M. L. (Ed.) Evaluating seedling quality: principles, procedures, and predictive abilities of major tests Proceedings. Corvallis: Forest
Research Laboratory, Oregon State University. 1985. p. 59-71.

USDA (United States Department of Agriculture). Soil Taxonomy: a basic system of soil classification for making and interpreting soil surveys. 2. ed. Washington, DC.: USDA, 1999. 870 p.

WARD, J. S.; GENT, M. P. N.; STEPHENS, G. R. Effects of planting stock quality and browse protection-type on height growth of northern red oak and eastern white pine. Forest Ecology and Management, Amsterdam, v. 127, p. 205-216, 2000.

ZIDA, D. et al. Initial seedling morphological characteristics and field performance of two Sudanian savanna species in relation to nursery production period and watering regimes. Forest Ecology and Management, Amsterdam, v. 255, p. 2151-2162, 2008.

Ci. Fl., v. 21, n. 2, abr.-jun., 2011 